\title{
PERTURBATIONS PRESERVING ASYMPTOTICS OF SPECTRUM WITH REMAINDER ${ }^{1}$
}

\author{
A. G. RAMM
}

ABstract. Let $A$ be a compact linear operator on a Hilbert space $H$, $s_{n}(A)=\lambda_{n}^{1 / 2}\left(A^{*} A\right), Q$ be a linear operator, $|Q f| \leq c|A f|^{a}|f|^{1-a}, a>0$, $c>0, \forall f \in H$. Let

$$
s_{n}(A)=c n^{-r}\left\{1+O\left(n^{-q}\right)\right\}, \quad r, q>0, B=A(I+Q) .
$$

Then

$$
s_{n}(B)=s_{n}(A)\left\{1+O\left(n^{-\gamma}\right)\right\}, \quad \gamma=\min \left(q, \frac{r a}{1+r a}\right) .
$$

Some applications of this result to the spectral theory of elliptic operators are given.

1. Introduction. In many cases it is interesting to know when the perturbed and the unperturbed operators have the same spectral asymptotic behavior. This question was discussed in [1]. Here we give an estimate of the remainder in the asymptotic formulas. This estimate is sharp.

\section{Main result.}

THEOREM 1. Let $A$ be a compact linear operator on a Hilbert space $H, Q$ be a linear operator such that $|Q f| \leq c|A f|^{a}|f|^{1-a}, a>0, c>0$, here and below denotes various constants. Let $B=A(I+Q)$ and $I+Q$ be invertible. If $s_{n}(A)=$ $c n^{-r}\left(1+O\left(n^{-q}\right)\right), r, q>0$, then

$$
s_{n}(B)=s_{n}(A)\left\{1+O\left(n^{-\gamma}\right)\right\}, \quad \gamma=\min \left(q, \frac{r a}{1+r a}\right) .
$$

Proof. Let $U=A^{*} A, V \equiv B^{*} B=\left(I+Q^{*}\right) U(I+Q), M_{n}$ be the $n$ dimensional subspace such that the condition $f \perp M_{n}$ is equivalent to the condition $g \equiv(I+Q) f \perp L_{n}$, and $L_{n}$ is the linear span of the first $n$ eigenvectors of $U$. From the minimax variational principle it follows that

$$
\begin{aligned}
s_{n+1+m}^{2}(B) & =\lambda_{n+1+m}(V) \leq \sup _{\substack{f \perp L_{m} \\
f \perp M_{n}}} \frac{(V f, f)}{|f|^{2}} \leq \sup _{f \perp M_{n}} \frac{(U g, g)}{(g, g)} \cdot \sup _{f \perp L_{m}} \frac{(g, g)}{|f|^{2}} \\
& =s_{n+1}^{2}(A)\left\{1+\sup _{f \perp L_{m}}\left(\frac{2|Q f|}{|f|}+\frac{|Q f|^{2}}{|f|^{2}}\right)\right\} \\
& =s_{n+1}^{2}(A)\left\{1+O\left(s_{m}^{a}(A)\right)\right\} .
\end{aligned}
$$

Received by the editors February 6, 1981 and, in revised form, April 27, 1981. The contents of this paper were presented at the 88th annual meeting of the Society (Notices Amer. Math. Soc. 29 (1982), p. 60).

1980 Mathematics Subject Classification. Primary 47A55, 47A10, 35P20.

${ }^{1}$ Supported by AFOSR 800204. 
Here we used the estimate

$$
\sup _{f \perp L_{m}} \frac{|Q f|}{|f|} \leq c \sup _{f \perp L_{m}} \frac{|A f|^{a}}{|f|^{a}} \leq c_{1} s_{m}^{a}(A) .
$$

Since $U=\left(I+S^{*}\right) V(I+S), I+S \equiv(I+Q)^{-1}, S=-(I+Q)^{-1} Q$, similar argument shows that

$$
s_{n+1+2 m}^{2}(A) \leq s_{n+1+m}^{2}(B)\left\{1+O\left(s_{m}^{a}(B)\right)\right\}
$$

One should use in the derivation of (3) $M_{n}^{\prime}$ instead of $M_{n}$, and the inequality $|S f| \leq c|Q f| \leq c_{1}|A f|^{a}|f|^{1-a} \leq c_{2}|B f|^{a}|f|^{1-a}$. Here $M_{n}^{\prime}$ is so chosen that the condition $f \perp \bar{M}_{n}^{\prime}$ is equivalent to the condition $g^{\prime} \equiv(I+S) f \perp L_{n}^{\prime}$ and $L_{n}^{\prime}$ is the linear span of the first $n$ eigenvectors of $V$. Since $s_{n}(A) \rightarrow 0$, and $s_{n}(B) \rightarrow 0$ it follows from (2) and (3) that $s_{n}(B) \cdot s_{n}^{-1}(A) \rightarrow 1$ as $n \rightarrow \infty$ and (1) holds. Indeed, take $m n^{-1}=n^{-x}$. Then $s_{m}^{a}(A)=O\left(n^{-r a(1-x)}\right)$ and $s_{n+m+1}(A) \cdot s_{n}^{-1}(A)=$ $1+O\left(n^{-x}\right)+O\left(n^{-q}\right)$ as $n \rightarrow \infty$ because $s_{n}(A)=c n^{-r}\left\{1+O\left(n^{-q}\right)\right\}$. Therefore

$$
\gamma=\min (q, x, r a(1-x))=\min \left(q, \frac{r a}{1+r a}\right) .
$$

Below $B \in R_{b}$ means that the root system of $B$ forms a Riesz basis with brackets of $H$ (see [2, p. 284] for definition).

THEOREM 1'. Let $B=A(I+Q), A>0, \lambda_{n}(A)=c n^{-r}\left(1+O\left(n^{-q}\right)\right), r, q>0$, $|Q f| \leq c\left|A^{b} f\right|, b>0, \operatorname{Ker}(I+Q)=\{0\}$. If $r b \geq 1$, then $B \in R_{b}$.

Proof. One has

$$
\begin{gathered}
B^{-1}=(I+Q)^{-1} A^{-1}=A^{-1}-(I+Q)^{-1} Q A^{-1} \\
\left|(I+Q)^{-1} Q A^{-1} f\right| \leq c_{1}\left|Q A^{-1} f\right| \leq c_{2}\left|A^{-(1-b)} f\right| \\
\lambda_{n}\left(A^{-1}\right)=c^{-1} n^{r}\left\{1+O\left(n^{-q}\right)\right\}
\end{gathered}
$$

From [2, p. 286, Theorem 1] it follows that $r b \geq 1$ implies $B^{-1} \in R_{b}$. Therefore $B \in R_{b}$.

Theorem 1 from [2] is stated in $§ 3$ below as Proposition 1.

REMARK 1. Estimate (1) is close to sharp: for the elliptic operators in $L^{2}(D)$ (in $\S 3$ below) the remainder is of order given in Theorem 1.

REMARK 2. Let $L$ be a closed densely defined linear operator on $H$ and $A=$ $L^{-1}$ be compact. Let $M$ be a linear operator $D(M) \supset D(L)$ and $T=M L^{-1}$ be compact. Then $B \equiv(L+M)^{-1}=A(I+T)^{-1}=A(I+Q), Q \equiv-(I+T)^{-1} T$. If $s_{n}(A)=c n^{-r}\left\{1+O\left(n^{-q}\right)\right\}$, and $|Q f| \leq c|A f|^{a}|f|^{1-a}, a>0$, then Theorem 1 is applicable. If one defines $s_{n}(L)=s_{n}^{-1}\left(L^{-1}\right)$ then $s_{n}(B)=s_{n}(A)\left\{1+O\left(n^{-\gamma}\right)\right\}$, i.e. $s_{n}(L+M)=s_{n}(L)\left\{1+O\left(n^{-\gamma}\right)\right\}$.

REMARK 3. The conclusion of Theorem 1 and its proof are valid also in the case when $B=(I+Q) A$ and $\left|Q^{*} f\right| \leq c|A f|^{a}|f|^{1-a}$. It follows from the equality $s_{n}(B)=s_{n}\left(B^{*}\right)$. In applications both estimates $|Q f| \leq c|A f|^{a}|f|^{1-a}$ and $\left|A^{*} f\right| \leq$ $c|A f|^{a}|f|^{1-a}$ are usually available.

\section{Applications to difierential operators.}

1. Let $L$ be an elliptic selfadjoint operator with smooth coefficients on $H=$ $L^{2}(D)$, where $D \subset \mathbf{R}^{d}$ is a bounded domain with a smooth boundary. Let $l=$ ord $L, m=\operatorname{ord} M, m<l$, where $M$ is some differential operator with smooth 
coefficients. Without loss of generality we assume that $L^{-1}$ and $(L+M)^{-1}$ exist. Let us define $s_{n}(L)=s_{n}^{-1}\left(L^{-1}\right)$. Then $s_{n}(L)=c n^{l / d}\left\{1+O\left(n^{-d^{-1}}\right)\right\}$.

$$
s_{n}(L+M)=s_{n}(L)\left\{1+O\left(n^{-\gamma}\right)\right\}, \quad \gamma=\min \left\{d^{-1}, \frac{l-m}{l-m+d}\right\}
$$

as $n \rightarrow \infty$. Indeed, $(L+M)^{-1}=\left(I+L^{-1} M\right)^{-1} L^{-1}=(I+Q) L^{-1}, Q=$ $-\left(I+L^{-1} M\right)^{-1} L^{-1} M$ and

$$
|Q f| \leq c\left|L^{-1} f\right|^{1-a}|f|^{a}, \quad a=m l^{-1} .
$$

The last inequality is an interpolation one which will be discussed in detail later. If $L^{-1}=A$, then (5) becomes

$$
|Q f| \leq c|A f|^{1-a}|f|^{a}
$$

and from Theorem 1 it follows that $s_{n}\left\{(L+M)^{-1}\right\}=s_{n}\left(L^{-1}\right)\left\{1+O\left(n^{-\gamma}\right)\right\}$, $\gamma=\min \left\{d^{-1},(l-m) /(l-m+d)\right\}$. This is equivalent to (4). Let us now explain (6). If $A>0$ then

$$
\left|A^{b} f\right| \leq|A f|^{b}|f|^{1-b}, \quad f \in D(A), 0<b<1 .
$$

Indeed, by Hölder inequality

$$
\begin{aligned}
\left|A^{b} f\right|^{2} & =\int_{0}^{\infty} t^{2 b} d\left(E_{t} f, f\right) \\
& \leq\left(\int_{0}^{\infty} t^{2} d\left(E_{t} f, f\right)\right)^{b}\left(\int_{0}^{\infty} d\left(E_{t} f, f\right)\right)^{1-b}=|A f|^{2 b} \cdot|f|^{2(1-b)} .
\end{aligned}
$$

This is equivalent to (7). Inequality (6) is a particular case of (7) with $L^{-1}=A$, $b=1-a$. Note that $\left|L^{-1} M f\right| \leq c\left|L^{-(1-a)} f\right|=c\left|A^{1-a} f\right|$. Let us formulate the result.

THEOREM 2. Let $L$ be an elliptic selfadjoint operator, ord $L=l$, on $H=L^{2}(D)$, where $D \subset \mathbf{R}^{d}$ is a bounded domain with a smooth boundary. Let $M$ be a differential operator ord $M=m<l$. Assume that the coefficients of $L$ and $M$ are smooth. Then

$$
s_{n}(L+M)=s_{n}(L)\left\{1+O\left(n^{-\gamma}\right)\right\}, \quad \gamma=\min \left\{d^{-1},(l-m)(l-m+d)^{-1}\right\} .
$$

REMARK 4. Under the assumptions of Theorem 2 the root system of the operator $L+M$ forms a Riesz basis of $H=L^{2}(D)$ with brackets if $l-m \geq d$.

This follows from Proposition 1.

PROPOSITION 1. Let $L=L^{*}>0$ be an operator with a discrete spectrum $\lambda_{j}=$ $c j^{p}+O\left(j^{p_{1}}\right), p>0, p_{1}<p$, and $|M u| \leq c\left|L^{a} u\right|, a<1 \forall u \in H$. Let $p(1-a) \geq 1$. Then the root system of $L+M$ forms a Riesz basis of $H$ with brackets.

A proof is given in [2]. The condition $l-m \geq d$ in Remark 1 guarantees that $p(1-a) \geq 1$. Indeed for the elliptic operator $L$ of order $l$ it is known that $p=l d^{-1}$. From the argument given in $\S 3$ it follows that $a=m l^{-1}$. Therefore $p(1-a)=d^{-1}\left(1-m l^{-1}\right)=(l-m) d^{-1} \geq 1$ because $l-m \geq d$. 
4. Bibliographical remarks.

1. If $\left|A^{-a} Q\right| \leq c$, or $|Q f| \leq c\left|A^{a} f\right|, a<1$, then it is often possible to derive the inequality used in Theorem 1: $|Q f| \leq c|A f|^{a}|f|^{1-a}$. For example this is so if $A$ satisfies the inequality

$$
\begin{array}{r}
\left|A^{\beta} f\right| \leq c(\alpha, \beta, \gamma)\left|A^{\gamma} f\right|^{(\beta-\alpha) /(\gamma-\alpha)} \cdot\left|A^{\alpha} f\right|^{(\gamma-\beta) /(\gamma-\alpha)}, \\
\alpha<\beta<\gamma, f \in D\left(A^{\gamma}\right) .
\end{array}
$$

This inequality is satisfied e.g. if $\left|(A+s I)^{-1}\right| \leq c(1+s)^{-1}$, $s>0$. If $\left|(A+s I)^{-1}\right| \leq c s^{-1}, \alpha<\beta<\gamma \leq 1$, and $\left.f \in \overline{D(} A^{\gamma}\right)$ then (*) holds. If $A=$ $A^{*}>0$ then $c(\alpha, \beta, \gamma)=1$. Inequality $(*)$ is closely connected with the theory of fraction powers of linear operators. In [3] one can find the related results and references.

2. In [4] the following theorem was proved: if $A$ and $T$ are compact linear operators on $H$ and for some $r>0 \lim s_{n}(A) n^{r}=c, \lim s_{n}(T) n^{r}=0$ as $n \rightarrow \infty$, then $\lim s_{n}(A+T) n^{r}=c$ as $n \rightarrow \infty$.

\section{REFERENCES}

1. A. G. Ramm, Perturbations preserving asymptotics of spectrum, J. Math. Anal. Appl. 76 (1980), 10-17.

2. - Theory and applications of some new classes of integral equations, Springer, New York, 1980.

3. S. G. Krein, Linear differential equations in Banach space, Transl. Math. Monos., Vol. 29, Amer. Math. Soc., Providence, R. I., 1972.

4. Ky Fan, Maximum properties and inequalities for the eigenvalues of completely continuous operators, Proc. Nat. Acad. Sci. U.S.A. 37 (1951), 760-766. 48109

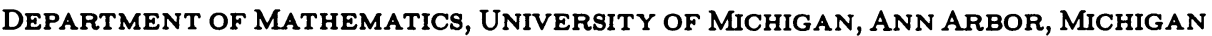

Current address: Department of Mathematics, Kansas State University, Manhattan, Kansas 66506 